\title{
Effect of BDNF and Adipose Derived Stem Cells Transplantation on Cognitive Deficit in Alzheimer Model of Rats
}

\author{
Parvin Babaei ${ }^{1,2}$, Bahram Soltani Tehrani ${ }^{1,3}$ \\ ${ }^{1}$ Cellular and Molecular Research Center, Guilan University of Medical Science, Rasht, Iran \\ ${ }^{2}$ Department of Physiology, Guilan University of Medical science, Rasht, Iran \\ ${ }^{3}$ Department of Pharmacology, Guilan University of Medical science, Rasht, Iran \\ Email: p_babaei@gums.ac.ir, bahram_s_t@yahoo.com
}

Received August 29, 2012; revised September 29, 2012; accepted October 19, 2012

\begin{abstract}
In this study, the potential for recovery mediated by co-treatment of brain-derived neurotrophic factor (BDNF) and adipose tissue derived stem cells (ASCs) on functional recovery after Ibotenic acid (Ibo) lesion of the nucleus basalis magnocellularis (NBM) was examined. Ibotenic acid was injected bilaterally into the NBM of experimental rats, then the animals received treatments as follows: ASCs $\left(500 \times 10^{3}\right)$, BDNF $(5 \mathrm{ug} / \mathrm{ul})$ and a combination of BDNF and ASCs. Two months after the treatment, cognitive recovery was assessed by the Morris water-maze. These results showed that ASCs transplantation may have therapeutic value in disease and conditions that result in memory loss, and co-treatment with BDNF doesn't offer more efficacious cognitive function.
\end{abstract}

Keywords: Alzheimer's Disease; Adipose Stem Cells; Brain-Derived Neurotrophic Factor; Learning and Memory

\section{Introduction}

Alzheimer's disease (AD) is a progressive dementia associated with cholinergic cell deterioration in the nucleus of Meynert, which results in the loss of cognitive functions $[1,2]$. In $\mathrm{AD}$, the severity of cognitive deficits correlates with synaptic loss in cholinergic neurons supplying neocortex and hippocampus [3].

Although adult brain has limited regenerative capability, to devise a method for manipulating and reimplanting neural stem cells is difficult. Stem cell therapy is one of the most interesting approaches for the treatment of neurodegenerative diseases. Our previous work showed that infusion of bone marrow mesenchymal stem cells in Alzheimer model of rats lead to improve learning and memory ability [4]. Recent studies have raised the possibility that adipose derived stem cells (ASCs) could be a good candidate for brain repair, due to their accessibility [5], and potency to differentiate into neurons [6,7]. However the main problem in stem cells transplantation strategy is how to improve cell survival in vivo. It has been known that the migration of stem cells is controlled by a set of special genes introducing the neurotrophic growth factors [8]. Studies show that BDNF, a member of the neurotrophin family, which is wildly expressed in adult brain areas, prevents neuronal degeneration during development [9] and promotes in vivo neurogenesis in adult forebrain
[10]. A new strategy on manipulating components of the niche that facilitates cross-talk between stem cells and the dysfunctional brain may offer more efficacious neurotransplantation [11]. The hypothesis that BDNF promotes survival and differentiation of grafted neural stem cells $[12,13]$ triggered us to investigate whether the cotreatment of BDNF with adipose stem cells increases the effects of transplanted cells in restoring cognitive deficit. Since the ultimate goal for cell therapy is functionality and few studies have examined a cognitive endpoint, here we focused on changes in learning and memory recovery.

\section{Materials \& Methods}

\subsection{Animals}

Fifty-seven male Wistar rats weighing 250 - $300 \mathrm{~g}$ were used for this study. Animals were housed with free access to food and water in a 12-h light/dark cycle and constant temperature. All procedures concerning animal care were in accordance with Guilan University of Medical Sciences ethical committee article (DEC No. 2719).

\subsection{Surgical Procedures \& Behavioral Test}

To establish the AD animal model, we infused Ibotenic acid into the nucleus basalis magnocellularis. On the day of surgery, the animals were anesthetized with ketamine/ 
xylazine $(50 \mathrm{mg} / \mathrm{kg}$, i.p.) and placed in a computerized stereotaxic apparatus (Neurostar, Germany). The incisor bar was set at: $-1.14 \mathrm{~mm}$ posterior and $\pm 2.46 \mathrm{~mm}$ lateral to the bregma and 7.9 below the top of the skull to reach the nucleus basalis magnocellularis [14], then guide cannula was implanted bilaterally for further infusions. Rats received bilateral infusions of $0.5 \mathrm{ul}$ or Ibotenic acid (10 ug/ul) using a 5 ul Hamilton syringe. After 14 days, rats were tested in the Morris Water Maze (MWM) in order to test learning ability. Animals who showed memory impairment were distributed into 4 groups: IBO + BDNF $(5 \mathrm{ug} / \mathrm{ul}), \mathrm{IBO}+\mathrm{ASCs}\left(500 \times 10^{3}\right.$ cells $), \mathrm{IBO}+(\mathrm{ASCs} /$ BDNF) and IBO+PBS. The control intact animals received only PBS. BDNF infusion was repeated twice for two weeks after the initiation of treatment in BDNF-receiving groups.

The Morris water maze [15] consisted of a black pool (148 cm diameter) filled with water $\left(26^{\circ} \mathrm{C} \pm 2^{\circ} \mathrm{C}\right)$. A circular black platform was submerged $2 \mathrm{~cm}$ below the water surface, in the middle of the target quadrant. The behavior of the rats in the pool could be tracked with a camera connected to Ethovision system (Noldus, EX 6.1, Netherlands) allowing us to measure swim speed, distance and latency to find the platform. Rats were trained with a protocol of four trials per day, with an interval of $20 \mathrm{~min}$, for 5 consecutive days. A probe trial was administered on the fifth day, when each subject was placed into the water diagonally opposite the target quadrant, and allowed $90 \mathrm{~s}$ to search the water, from which the platform had been removed.

\subsection{Adipose Stem Cells Isolation and Culture}

Adipose tissue was obtained from the abdomen. One gram adipose tissue was incubated with $1.5 \mathrm{mg}$ collagenase type II in $10 \mathrm{ml}$ saline at $37^{\circ} \mathrm{C}$ for one hour. Digested adipose tissues were centrifuged for ten minutes at $1500 \mathrm{rpm}$, and pellets were washed with $10 \mathrm{ml}$ and plated in $25 \mathrm{ml}$ cultured flask containing DMEM, 10\% FBS and antibiotics. Then cells were incubated at $37^{\circ} \mathrm{C}$ in a humidified atmosphere containing $95 \%$ air and $5 \% \mathrm{CO}_{2}$. On reaching confluency, the adherent cells were detached by $0.05 \%$ trypsin and $0.02 \%$ EDTA for $5 \mathrm{~min}$ at $37^{\circ} \mathrm{C}$, harvested and washed with DMEM and 10\% FBS and finally resuspended in complete medium. After 2 - 3 passages, the morphologically homogeneous population of ASCs was analyzed for the expression of cell surface molecules using histology staining. Flow cytometry test was used for detecting stem cells markers of CD44, CD 105 , CD90 according to standard protocols [5]. Cell suspensions with viability more than $90 \%$, were made at a density of $500 \times 10^{3}$ cells $/ \mu 1$ and were kept on ice to optimize cell viability until infusion.

\subsection{Statistical Analysis}

The data is expressed as means \pm SEM. Group differences in the escape latency in the Morris water maze probe task were analyzed using one-way analysis of variance (ANOVA) followed by Tukey's post hoc test. The repeated ANOVA measure for multiple group comparison was used to analyze group differences of the data collected during the training days.

\section{Results}

Adipose stem cells were successfully culture-expanded and a morphologically homogeneous population of fibroblast-like cells was seen after 14 days. Flow cytometry analysis showed uniformly positive stem cells for CD44, CD105, CD90 as shown in Figure 1.

Since the ultimate measure of stem cells transplantation into the brain is functionality, we tested animals two months after transplantation. As seen in Figure 2, there was no initial difference between treated animals during the first days of acquisition in the Morris water maze.

Treated rats acquired the task rapidly in all groups, and latency to escape diminished over time $(p<0.001)$. Since the experimental groups didn't differ in swim speed (Ibo: $22 \pm 0.6 \mathrm{~cm} / \mathrm{s}$, cell/BDNF: $22.7 \pm 0.4 \mathrm{~cm} / \mathrm{s}$, ASCs: $21.8 \pm$ $0.93, F(4,170)=0.85, p>0.05)$, we used latency to find the platform as an indicator of learning performance. The improvement in acquisition was found in ASCs-transplanted group across trials $\left(\mathrm{F}_{9,387}=91.3 ; \mathrm{p}<0.0001\right)$. Cell transplanted rats spent shorter time $(11.3 \pm 0.9 \mathrm{sec}$; Figures 3 and 5) to reach the platform in probe test, and also spent more time in the target quadrant $(25 \pm 1.34 \mathrm{sec}$; Figure 4) compared to other treated groups, except unlesioned control group.

There was no statistical difference in the ability of learning and memory between BDNF and Ibo + PBS ( $p=$ 0.13 ); however, the BDNF group showed better performance (Figure 2). Although water maze acquisition was improved by adipose stem cells transplantation, but none of the treated groups reached to the level of the naive group (Figure 2).

\section{Discussion}

In the present study, the transplanted group showed an improvement in learning performance, indicating effectiveness of ASCs in restoring learning capability of AD animals two months after transplantation. This finding is in agreement with the results of similar studies on bone marrow mesenchymal stem cells [4], neural stem cells $[13,16]$, and umbilical cord blood stem cells transplantation in $\mathrm{AD}$ animals [17]. Although the mechanisms of recovery are not completely understood, a first explanation could be the capacity of ASCs to dif ferentiate into 

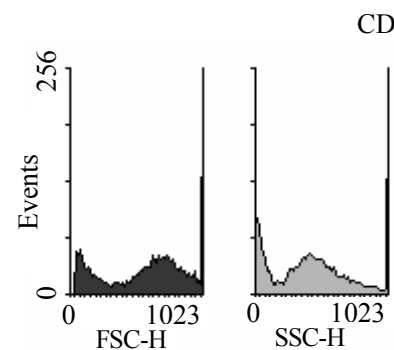

D 105
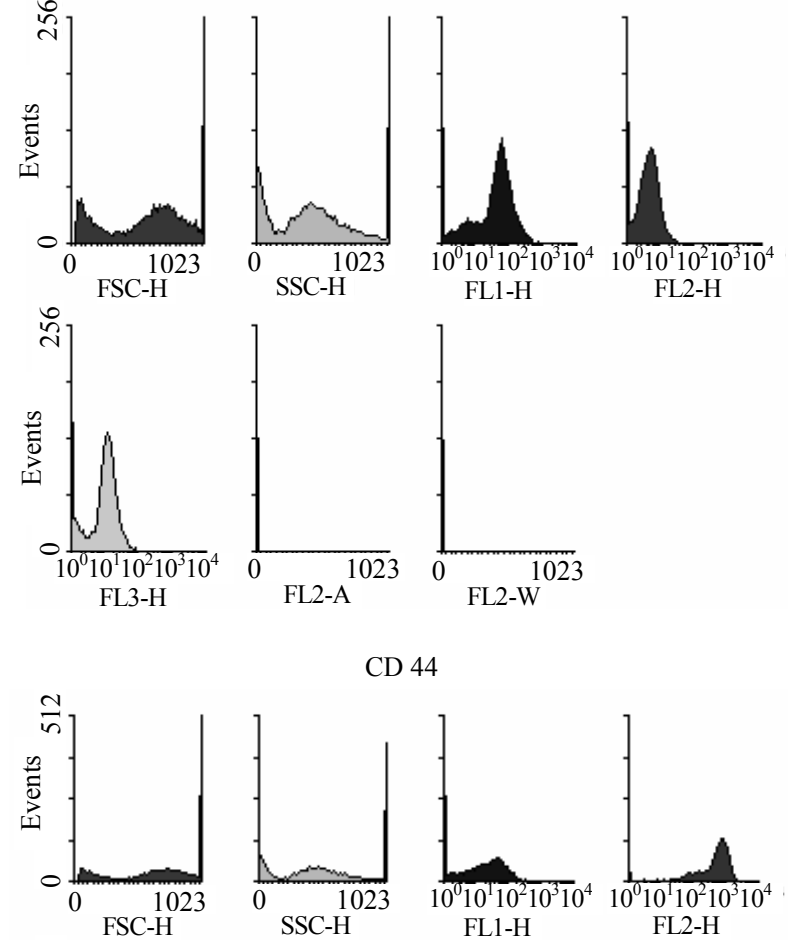

\section{44}
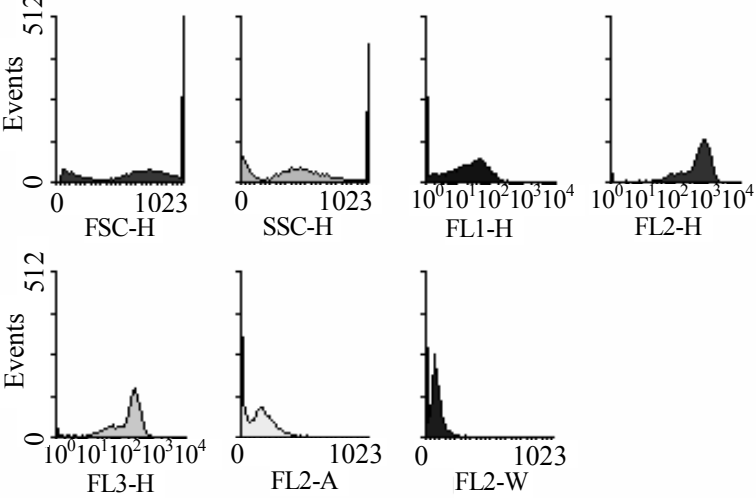

CD 90
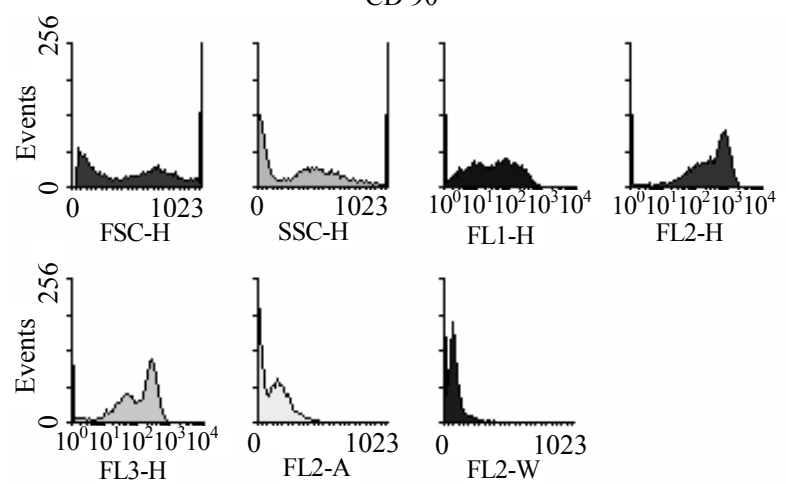

Figure 1. Flow cytometric analysis of ASCs indicated cell surface phenotype characteristic CD44, CD90, CD105.

the new cholinergic neurons to compensate cholinergic deficit. The potency of these cells to differentiate into the neuron has been reported in previous studies $[3,6]$. However we cannot exclude the possibility that stem cells may provide therapeutic effects by keeping the integrity of neurons through exerting protective chaperone effect $[12,18]$ or neurochemical modulation.

Moreover, we showed that infusion of recombinant

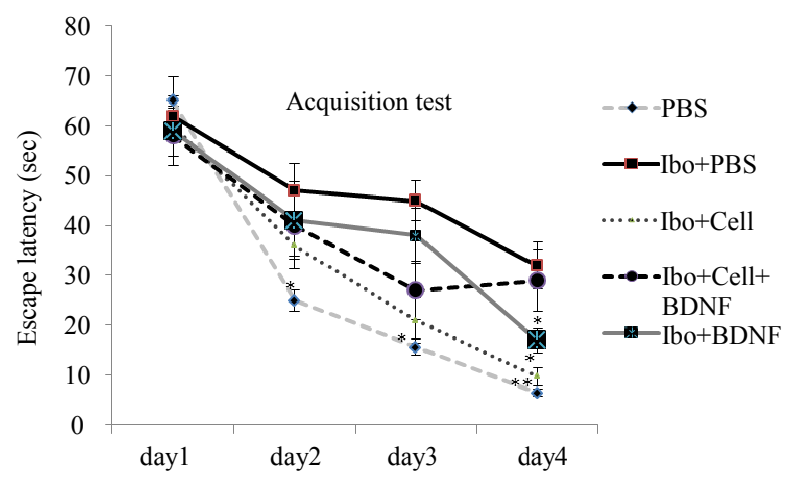

Figure 2. The ability of spatial learning in MWM. PBS control (n = 13), Ibo + PBS (n = 10), IBO + BDNF ( $=8)$, IBO + ASCs (n = 14), IBO + (ASCs + BDNF, n = 8) ${ }^{*} \mathrm{p}<0.01,{ }^{* *} \mathrm{p}$ $<0.001$ compared with Ibo + group.

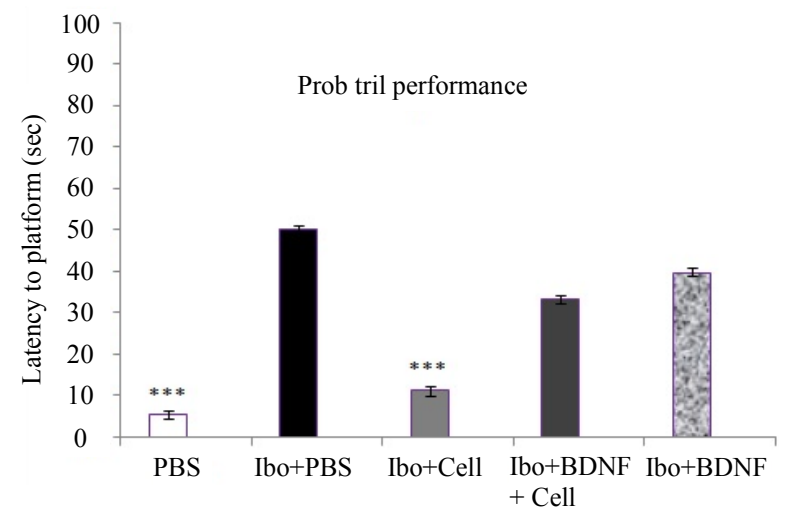

Figure 3. Performance of animals in prob trial. Means and standard errors of the latency to find the platform, in sec are depicted PBS control $(n=13)$, Ibo + PBS $(n=10)$, IBO + BDNF (n = 8), IBO + ASCs $(n=14)$, IBO + (ASCs/BDNF, $\mathrm{n}=8){ }^{* *} \mathrm{p}<0.001$ compared with Ibo-group.

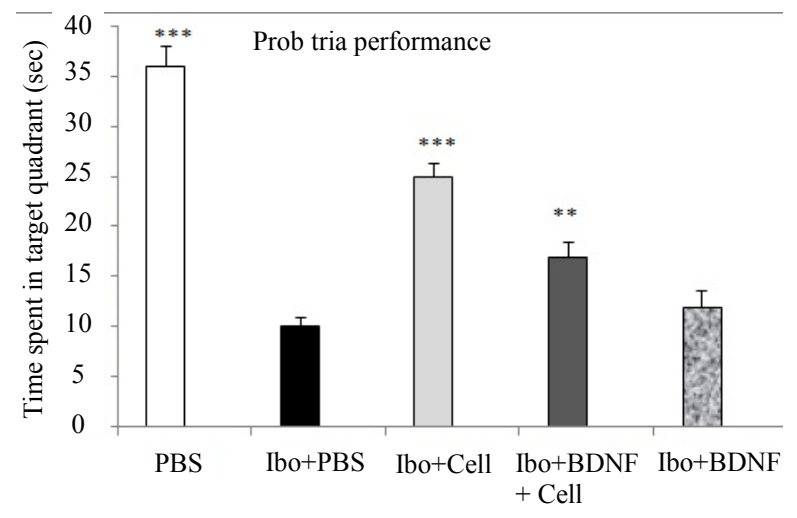

Figure 4. The ability of spatial learning in MWM. PBS control (n = 13), Ibo + PBS (n = 10), IBO + BDNF (n = 8), IBO + ASCs (n = 14), IBO + (ASCs + BDNF, $n=8){ }^{*} \mathrm{p}<0.01,{ }^{* *} \mathrm{p}$ $<0.001$ compared with Ibo + group.

BDNF into the NBM, 14 days afte the lesion, slightly reverses the deficit in memory caused by Ibo. It has been documented that BDNF is implicated in learning and memory $[19,20]$. Several investigators have reported pro- 

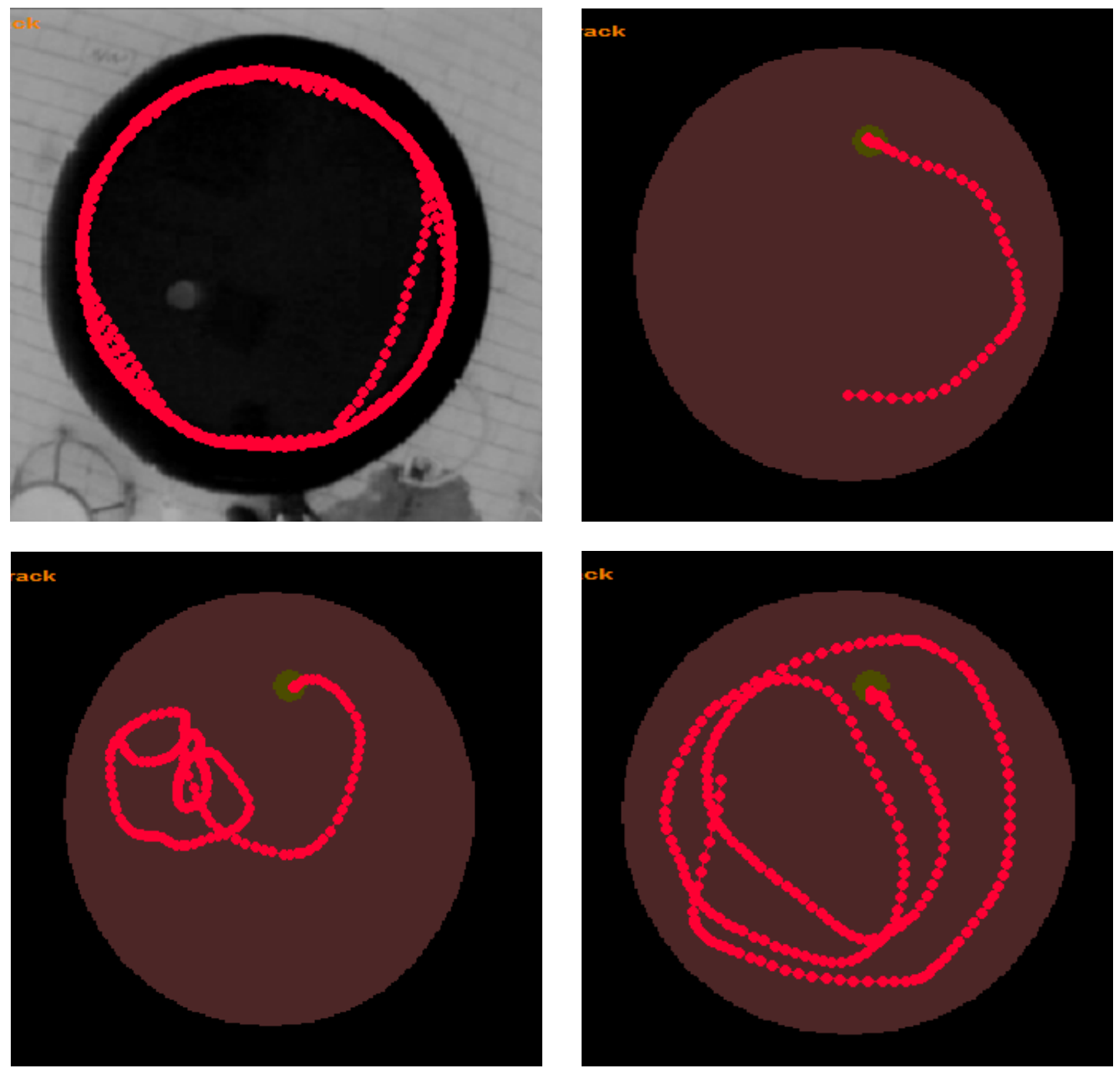

Figure 5. Shows a sample of computer tracking from probe trial (90 sec duration). Top left: Ibotenic acid, top right: ASCs transplanted, down left: BDNF and down right: ASCs + BDNF. The rat of Ibo group swims in a concentric pattern, whereas cell treated group swims in a short direct pattern toward the hidden platform.

tective and anti-apoptotic effects of BDNF on neural cells $[9,12,21]$ and that it induces neurogenesis [22]. It is possible that BDNF induces the survival of some but not all of the degenerating cholinergic neurons in the NBM.

We initially assumed that adding BDNF to stem cells will increase learning and memory performance. Much to our surprise, the present study shows that adding BDNF to ASCs leads to improvement in learning ability, but in a lesser extent compared to cells alone. Our results appear to contradict results of Xuan [13], who reported that BDNF improves the effects of neural stem cells on the rat model of Alzheimer's disease. The reasons for this discrepancy might be the source of stem cells which in their studies was neural stem cells, and also method of lesion, which was unilateral fimbria-fornix lesioning which causes axonal damage [23], whereas IBO induces neuronal necrosis which is confined to somata of neurons, without affecting on myelinated axons or blood vessels [24].

To explain why adding BDNF to stem cells didn't lead to an enhancement in learning performance compared to stem cells infusion, one can point to the concentration of the BDNF. There are studies showing that Brain-derived neurotrophic factor in high concentration might induce adverse effects. Although we didn't measure the BDNF levels in NBM after the transplantation, the possible release of BDNF by injured brain [2], glia cells [25] and also transplanted ASCs (Kang et al., 2003; McCoy et al., 2008), might lead to increase in the level of BDNF, and this neurothrophine, in high concentration causes learning deficits $[26,27]$. BDNF plays different roles in learning and memory depending on receptors; the receptor P75NTR, involves in, LTD and cell death, however Trk $\beta$ is responsible for LTP and survival of neurons [26].

To answer this question that how much stem cells ther- 
apy is efficient for Alzheimer treatment, we should mention that although enhancement of learning ability was achieved after the ASCs transplantation, but none of the treated groups reached the control levels performance. This indicates that adding stem cells to neural network is not sufficient to restore learning and memory completely. It is well known that the learning process, as a complex phenomenon in CNS, needs an orchestrated chain of translational and transcriptional events in functional synapses in order to encode, store, and recall information appropriately [20].

One limitation of this study is not being able to assess the fate of the transplanted stem cells and follow up the functional outcome of grafted cells over three months. Although transplanted cells take over a month in vivo to develop, the electrophysiological responsiveness of mature neurons form long projecting axons to the forebrain, but it takes at least 3 months for a memory to manifest $[21,28]$.

\section{Discussion}

Our results showed that ASCs treatment significantly increased learning and memory ability in Alzheimer model of rats. Addition of BDNF to ASCs doesn't improve the effectiveness of cells in restoring cognitive function.

In a clinical point of view, and considering less ethical problems and ease of accessibility, adipose stem cells could be a valuable therapeutic tool in treating patients suffering from neurodegenerative diseases. More broadly, this study supports the view that manipulating components of the niche affects on cross-talk between stem cells and the dysfunctional brain, but not always bring efficacious neuro transplantation outcome.

\section{Acknowledgements}

This work was supported by grants from the Research Council of Guilan University of Medical Sciences.

\section{REFERENCES}

[1] C. Geula, N. Nagykery, A. Nicholas and C. K. Wu, "Cholinergic Neuronal and Axonal Abnormalities Are Present Early in Aging and in Alzheimer Disease," Journal of Neuropathology \& Experimental Neurology, Vol. 67, No. 4, 2008, pp. 309-318. doi:10.1097/NEN.0b013e31816a1df3

[2] A. Swarowsky, L. Rodrigues, R. Biasibetti, M. C. Leite, L. F. de Oliveira, L. M. de Almeida, C. Gottfried, J. A. Quillfeldt, M. Achaval and C. A. Goncalves, "Glial Alterations in the Hippocampus of Rats Submitted to Ibotenic-Induced Lesion of the Nucleus Basalis Magnocellularis," Behavioural Brain Research, Vol. 190, No. 2, 2008, pp. 206-211. doi:10.1016/j.bbr.2008.02.039

[3] K. L. Davis, R. C. Mohs, D. Marin, D. P. Purohit, D. P.
Perl, M. Lantz, G. Austin and V. Haroutunian, "Cholinergic Markers in Elderly Patients with Early Signs of Alzheimer Disease," Journal of the American Medical Association, Vol. 281, No. 15, 1999, pp. 1401-1406. doi:10.1001/jama.281.15.1401

[4] P. Babaei, B. Soltani Tehrani and A. Alizadeh, "Transplanted Bone Marrow Mesenchymal Stem Cells Improve Memory in Rat Models of Alzheimer's Disease," Stem Cells International, Vol. 2012, 2012, pp. 1-8. doi: $10.1155 / 2012 / 369417$

[5] M. J. Lopez and N. D. Spencer, "In Vitro Adult Rat Adipose Tissue-Derived Stromal Cell Isolation and Differentiation," Methods in Molecular Biology, Vol. 702, 2011, pp. 37-46. doi:10.1007/978-1-61737-960-4 4

[6] S. K. Kang, D. H. Lee, Y. C. Bae, H. K. Kim, S. Y. Baik and J. S. Jung, "Improvement of Neurological Deficits by Intracerebral Transplantation of Human Adipose TissueDerived Stromal Cells after Cerebral Ischemia in Rats," Experimental Neurology, Vol. 183, No. 2, 2003, pp. 355366. doi:10.1016/S0014-4886(03)00089-X

[7] M. K. McCoy, T. N. Martinez, K. A. Ruhn, P. C. Wrage, E. W. Keefer and M. G. Tansey, "Autologous Transplants of Adipose-Derived Adult Stromal (ADAS) Cells Afford Dopaminergic Neuroprotection in a Model of Parkinson's Disease," Experimental Neurology, Vol. 210, No. 1, 2008, pp. 14-29. doi:10.1016/j.expneurol.2007.10.011

[8] P. Taupin and F. H. Gage, "Adult Neurogenesis and Neural Stem Cells of the Central Nervous System in Mammals," Journal of Neuroscience Research, Vol. 69, No. 6, 2002, pp. 745-749. doi:10.1002/jnr.10378

[9] C. M. Morshead and D. Vanderkooy, "Postmitotic Death Is the Fate of Constitutively Proliferating Cells in the Subependymal Layer of the Adult-Mouse Brain," The Journal of Neuroscience, Vol. 12, No. 1, 1992, pp. 249256.

[10] V. Pencea, K. D. Bingaman, S. J. Wiegand and M. B. Luskin, "Infusion of Brain-Derived Neurotrophic Factor into the Lateral Ventricle of the Adult Rat Leads to New Neurons in the Parenchyma of the Striatum, Septum, Thalamus, and Hypothalamus," The Journal of Neuroscience, Vol. 21, No. 17, 2001, pp. 6706-6717.

[11] V. Ourednik, J. Ourednik, Y. Xu, Y. Zhang, W. P. Lynch, E. Y. Snyder and M. Schachner, "Cross-Talk between Stem Cells and the Dysfunctional Brain Is Facilitated by Manipulating the Niche: Evidence from an Adhesion Molecule," Stem Cells, Vol. 27, No. 11, 2009, pp. 2846-2856. doi: $10.1002 /$ stem. 227

[12] T. Suzuki, S. Ooto, T. Akagi, K. Amemiya, Y. Igarashi, R. Mizushima and M. Takahashi, "Effects of Prolonged Delivery of Brain-Derived Neurotrophic Factor on the Fate of Neural Stem Cells Transplanted into the Developing Rat Retina," Biochemical and Biophysical Research Communications, Vol. 309, No. 4, 2003, pp. 843-847. doi:10.1016/j.bbrc.2003.08.076

[13] A. G. Xuan, D. H. Long, H. G. Gu, D. D. Yang, L. P. Hong and S. L. Leng, "BDNF Improves the Effects of Neural Stem Cells on the Rat Model of Alzheimer's Disease with Unilateral Lesion of Fimbria-Fornix," Neuroscience Letters, Vol. 440, No. 3, 2008, pp. 331-335. 
doi:10.1016/j.neulet.2008.05.107

[14] G. Paxinos and C.Watson, "The Rat Brain in Stereotaxic Coordinates," 6th Edition, Elsevier, Amsterdam, 2007. http://www.pricestube.com/export/9780123741219.pdf

[15] R. Morris, "Developments of a Water-Maze Procedure for Studying Spatial Learning in the Rat," Journal of Neuroscience Methods, Vol. 11, No. 1, 1984, pp. 47-60. doi:10.1016/0165-0270(84)90007-4

[16] M. Mizuno, K. Yamada, A. Olariu, H. Nawa and T. Nabeshima, "Involvement of Brain-Derived Neurotrophic Factor in Spatial Memory Formation and Maintenance in a Radial Arm Maze Test in Rats," The Journal of Neuroscience, Vol. 20, No. 18, 2000, pp. 7116-7121.

[17] H. J. Lee, J. K. Lee, H. Lee, J. W. Shin, J. E. Carter, T. Sakamoto, H. K. Jin and J. S. Bae, "The Therapeutic Potential of Human Umbilical Cord Blood-Derived Mesenchymal Stem Cells in Alzheimer's Disease," Neuroscience Letters, Vol. 481, No. 1, 2010, pp. 30-35. doi:10.1016/j.neulet.2010.06.045

[18] X. Chen, M. Katakowski, Y. Li, D. Lu, L. Wang, L. Zhang, J. Chen, Y. Xu, S. M. Gautam and M. Chopp, "Human Bone Marrow Stromal Cell Cultures Conditioned by Traumatic Brain Tissue Extracts: Growth Factor Production," Journal of Neuroscience Research, Vol. 69, No. 5, 2002, pp. 687-691. doi:10.1002/jnr.10334

[19] S. A. Heldt, L. Stanek, J. P. Chhatwal and K. J. Ressler, "Hippocampus-Specific Deletion of BDNF in Adult Mice Impairs Spatial Memory and Extinction of Aversive Memories," Molecular Psychiatry, Vol. 12, No. 7, 2007, pp. 656-670. doi:10.1038/sj.mp.4001957

[20] L. Tapia-Arancibia, E. M. Aliaga and S. Silhol, "New Insights into Brain BDNF Function in Normal Aging and Alzheimer Disease," Brain Research Reviews, Vol. 59, No. 1, 2008, pp. 201-220. doi:10.1016/j.brainresrev.2008.07.007

[21] B. Waldau, "Stem Cell Transplantation for Enhancement of Learning and Memory in Adult Neurocognitive Disorders," Aging and Disease, Vol. 1, No. 1, 2010, pp. 60-71.

[22] M. B. Luskin, V. Pencea, K. D. Bingaman and S. J. Wie- gand, "Infusion of Brain-Derived Neurotrophic Factor into the Lateral Ventricle of the Adult Rat Leads to New Neurons in the Parenchyma of the Striatum, Septum, Thalamus, and Hypothalamus," Journal of Neuroscience, Vol. 21, No. 17, 2001, pp. 6706-6717.

[23] S. D. Ginsberg and L. J. Martin, "Axonal Transection in Adult Rat Brain Induces Transsynaptic Apoptosis and Persistent Atrophy of Target Neurons," Journal of Neurotrauma, Vol. 19, No. 1, 2002, pp. 99-109. doi: $10.1089 / 089771502753460277$

[24] M. Eijkenboom, A. Blokland and F. J. van der Staay, "Modelling Cognitive Dysfunctions with Bilateral Injections of Ibotenic Acid into the Rat Entorhinal Cortex," Neuroscience, Vol. 101, No. 1, 2000, pp. 27-39. doi:10.1016/S0306-4522(00)00342-0

[25] H. Song, C. F. Stevens and F. H. Gage, "Astroglia Induce Neurogenesis from Adult Neural Stem Cells," Nature, Vol. 417, No. 6884, 2002, pp. 39-44. doi:10.1038/417039a

[26] C. Cunha, A. Angelucci, A. D'Antoni, M. D. Dobrossy, S. B. Dunnett, N. Berardi and R. Brambilla, "Brain-Derived Neurotrophic Factor (BDNF) Overexpression in the Forebrain Results in Learning and Memory Impairments," Neurobiology of Disease, Vol. 33, No. 3, 2009, pp. 358368. doi:10.1016/j.nbd.2008.11.004

[27] S. D. Croll, C. Suri, D. L. Compton, M. V. Simmons, G. D. Yancopoulos, R. M. Lindsay, S. J. Wiegand, J. S. Rudge and H. E. Scharfman, "Brain-Derived Neurotrophic Factor Transgenic Mice Exhibit Passive Avoidance Deficits, Increased Seizure Severity and in Vitro Hyperexcitability in the Hippocampus and Entorhinal Cortex," Neuroscience, Vol. 93, No. 4, 1999, pp. 1491-1506. doi:10.1016/S0306-4522(99)00296-1

[28] U. Englund, A. Bjorklund, K. Wictorin, O. Lindvall and M. Kokaia, "Grafted Neural Stem Cells Develop into Functional Pyramidal Neurons and Integrate into Host Cortical Circuitry," Proceedings of the National Academy of Sciences, Vol. 99, No. 26, 2002, pp. 17089-17094. doi:10.1073/pnas.252589099 